\title{
Análise epidemiológica e espacial dos casos de sífilis gestacional e congênita
}

\author{
Epidemiological and spatial analysis of cases of gestational and \\ congenital syphilis
}

Hayla Nunes da Conceição', Joseneide Teixeira Câmara², Beatriz Mourão Pereira²

DOI: 10.1590/0103-1104201912313

RESUMO O presente estudo teve como objetivo analisar o perfil epidemiológico e a distribuição espacial dos casos de sífilis gestacional e congênita. Trata-se de uma pesquisa descritiva, exploratória e retrospectiva, com abordagem quantitativa, realizada em Caxias (MA). Utilizaram-se os dados das fichas de investigação dos casos confirmados de sífilis gestacional e congênita do Sistema de Informação de Agravos de Notificação no período de 2013 a 2017. Com a tecnologia Global Positioning System (Sistema de Posicionamento Global), foram coletadas as coordenadas dos casos, e com o programa Quantum GIS, versão 1.7.0, os mapas foram construídos. Foram 149 casos de sífilis gestacional, apresentando crescimento de 73\% no período. As maiores prevalências ocorreram em mulheres jovens, pardas, com baixa escolaridade e donas de casa. Evidenciou-se maior frequência do diagnóstico no terceiro trimestre gestacional, com maior prevalência de sífilis primária. Foram 18 casos de sífilis congênita, com redução de 33\% na taxa de detecção e prevalência na idade entre 1 e 28 dias de vida. Na análise espacial, as zonas Oeste e Leste, periferia do município, concentraram o maior número de casos. Revelou-se frágil o manejo de investigação, tornando necessária a educação permanente para qualificar os profissionais, com o intuito de instituir a identificação precoce, o tratamento oportuno e o acompanhamento efetivo.

PALAVRAS-CHAVE Sífilis congênita. Transmissão vertical de doença infecciosa. Cuidado pré-natal.

ABSTRACT The aim of the present study was to analyze the epidemiological profile and spatial distribution of gestational and congenital syphilis cases. This is a descriptive, exploratory and retrospective research, with a quantitative approach, conducted in Caxias (MA). Data from case reports confirmed by gestational and congenital syphilis of the Notification Recording Information System from 2013 to December 2017 were used. With the Global Positioning System technology, case coordinates were collected, and with the Quantum GIS program, version 1.7.0, the maps were built. There were 149 cases of gestational syphilis, presenting a growth of $73 \%$ in the period. The highest prevalence occurred in young, brown women with low schooling and housewives. Higher frequency of diagnosis in the third gestational trimester was evidenced, with higher prevalence of primary syphilis. There were 18 cases of congenital syphilis, with a $33 \%$ reduction in detection rate and age prevalence between 1 and 28 days of life. In the spatial analysis, the West and East zones, outskirts of the municipality, concentrated the largest number of cases. It reveals the fragility in the research management, making it detrimental to permanent education, to qualify the professionals, in order to institute

1 Universidade Federal do Piauí (UFPI) - Teresina (PI), Brasil. haylanunes_cx@hotmail. com

2 Universidade Estadual do Maranhão (Uema) - São Luís (MA), Brasil.
KEYWORDS Syphilis, congenital. Infectious disease transmission, vertical. Prenatal care. 


\section{Introdução}

A sífilis é uma infecção causada pela bactéria Treponema pallidum (T. pallidum), que é transmitida por via sexual, transfusão sanguínea e através da transmissão vertical, quando a mãe com diagnóstico de sífilis não é tratada ou não realiza o esquema de tratamento adequadamente' ${ }^{1}$ É uma doença que apresenta tratamento acessível, efetivo e eficaz, mas ainda exibe altas taxas de incidência, representando um desafio para a saúde pública².

A taxa de transmissão vertical da sífilis, nas fases primárias e secundárias da doença, é de $70 \%$ a $100 \%$ nas gestantes que não recebem tratamento e/ou são tratadas inadequadamente, com redução nas fases latente e tardia. A infecção pela T. pallidum pode desencadear aborto espontâneo, malformações congênitas, natimorte ou morte perinatal em cerca de $40 \%$ das crianças infectadas ${ }^{2-4}$.

Segundo a Organização Mundial da Saúde (OMS), estima-se que, no mundo, ocorram anualmente cerca de 12 milhões de novos casos de sífilis, ressaltando que destes, 1,5 a 1,85 milhões dos registros encontrados são de gestantes, e que $50 \%$ delas têm filhos com resultados adversos devido às consequências da doença ${ }^{4}$.

O Brasil apresentou um aumento na taxa de incidência de sífilis gestacional e congênita, no período de 2010 a 2016, na qual a sífilis gestacional passou de 3,5 para 12,4 casos a cada mil nascidos vivos, e a sífilis congênita aumentou de 2,4 para 6,8 casos por mil nascidos vivos, um aumento de aproximadamente três vezes no número de casos ${ }^{5}$.

A região Nordeste ocupa o segundo lugar do ranking das regiões com maior número de casos de sífilis em gestantes no País, sendo o estado do Maranhão o que apresenta a maior taxa de incidência dessa região, com seis casos por mil nascidos vivos, concentrando as maiores taxas em São Luís, capital do estado, com 6,4\% dos casos, seguido pelos municípios de Imperatriz $(6,2 \%)$, Codó (5,6\%) e Caxias $(5,6 \%)^{5,6}$.

Assim, a intensificação da vigilância da infecção de sífilis em gestante é fundamental, e tem o objetivo de conhecer o estado sorológico e iniciar a terapêutica materna precocemente, possibilitando o planejamento e a avaliação das medidas de prevenção e controle, particularmente da transmissão vertical da T. pallidum? ${ }^{7}$.

No tocante aos dados epidemiológicos, considerando o crescente aumento de notificações de sífilis gestacional e consequentemente sífilis congênita no Nordeste e, principalmente, no Maranhão, é de fundamental importância conhecer o perfil das mulheres grávidas e crianças infectadas com sífilis, bem como detectar as áreas com maior foco para esses agravos e identificar as barreiras que comprometem o controle da sífilis em nível local, para que se possa adotar medidas de redução dessas infecções e propor medidas de intervenção efetivas ${ }^{5}$.

Nessa perspectiva, o objetivo do presente estudo foi analisar o perfil epidemiológico e a distribuição espacial dos casos de sífilis gestacional e congênita.

\section{Material e métodos}

Trata-se de uma pesquisa exploratória, descritiva, ecológica e retrospectiva, com abordagem quantitativa, realizada através da coleta dos dados fornecidos pela Vigilância Epidemiológica da Secretaria Municipal de Saúde do município de Caxias (MA), com as informações provenientes do Sistema de Informação de Agravos de Notificação (Sinan) e do Sistema de Informação sobre Nascidos Vivos (Sinasc).

A população do estudo foi composta por todos os casos confirmados de sífilis gestacional ( $n=149)$ e congênita $(n=18)$ no período de janeiro de 2013 a dezembro de 2017, residentes no município de Caxias (MA), e que estavam registrados no Sinan. Foram excluídas as fichas de notificações duplicadas, que não apresentaram ano de notificação, com dados ilegíveis e os casos que, apesar de terem sidos notificados em Caxias (MA), não eram residentes no município.

As variáveis investigadas sobre o perfil das gestantes com a infecção foram: número de 
casos de sífilis gestacional por ano de notificação, faixa etária, escolaridade, raça/cor, ocupação, período gestacional do diagnóstico, classificação clínica, realização de teste treponêmico no pré-natal, realização de teste não treponêmico no pré-natal, titulação, esquema de tratamento prescrito à gestante, realização do tratamento do parceiro, esquema de tratamento prescrito ao parceiro e motivo do não tratamento do parceiro das gestantes sororreagentes para sífilis.

Para a investigação da sífilis congênita foram analisadas as seguintes características referentes à mãe: idade, raça/cor, escolaridade, endereço residencial, realização do pré-natal, momento do diagnóstico da sífilis materna, adequação do tratamento para a sífilis materna e o tratamento do parceiro da gestante. Além disso, foram investigadas as características sociais, assistenciais e diagnósticas relativas aos casos de crianças, compreendendo: idade, sexo, raça/cor, endereço residencial, presença de sinais e sintomas, realização do teste não treponêmico (sangue periférico e líquor), radiografia de ossos longos, esquema de tratamento prescrito à criança e evolução do caso.

Para o cálculo da taxa de incidência da sífilis gestacional, utilizou-se o número de casos de sífilis detectados em gestantes residentes no município e foi realizada a divisão pelo número total de nascidos vivos de mulheres residentes no município de estudo, obtido no Sinasc e multiplicado por mil. Já o cálculo da incidência de casos de sífilis congênita foi realizado com a utilização do número de casos de sífilis congênita e o número de nascidos vivos, a partir da equação de incidência dos casos (número de casos novos da doença dividido pelo número de nascidos vivos e multiplicado por mil) ${ }^{8}$. Para o cálculo da redução percentual das taxas de incidência da infecção em ambos os grupos, entre o primeiro e último ano de estudo, realizou-se a subtração entre o valor inicial e o valor final, e dividiu-se o resultado pelo valor inicial e, posteriormente, multiplicou-se por cem.

Para a descrição do perfil epidemiológico dos casos confirmados das variáveis utilizadas para a pesquisa, através do programa Epi Info versão 3.5.1, realizaram-se os cálculos referentes à estatística descritiva, com a elaboração das frequências absolutas, que, em seguida, foram organizadas em tabelas e gráficos.

Na construção dos mapas, foram coletadas as coordenadas geográficas com auxílio do Global Positioning System (GPS) Essentials, no qual, conforme endereço residencial da ficha de notificação dos casos confirmados, eram feitas as marcações das coordenadas. Em seguida, na elaboração dos mapas, foi utilizado o programa o QGIS versão 2.18 Las Palmas - QGIS Brasil, que utilizou os dados das coordenadas coletadas e realizou a distribuição espacial através do mapa de Kernel, conforme a zonas que compõem o município: Oeste, Leste, Central, Norte e Sul.

Em consonância com o exigido pelas diretrizes e normas regulamentadoras da pesquisa envolvendo seres humanos, preconizadas na Resolução n ${ }^{\circ} 466 / 2012^{9}$, o projeto de pesquisa foi submetido ao Comitê de Ética em Pesquisa em Seres Humanos da Universidade Estadual do Maranhão, aprovado com o parecer $n^{\circ}$ 3.047 .824 .

\section{Resultados}

No período de 2013 a 2017, foram notificados 149 casos de sífilis gestacional e 18 casos de sífilis congênita no município. Identificouse um crescimento de $73 \%$ na incidência dos casos de sífilis gestacional, sobretudo em 2016 e 2017, enquanto a sífilis congênita apresentou um declínio significativo na incidência, com redução de $33 \%$ do número de casos, destacando o ano de 2015 com menor taxa de incidência (zero casos/mil nascidos vivos) (gráfico 1). 
Gráfico 1. Taxa de incidência de sífilis gestacional e congênita (por mil nascidos vivos) no município de Caxias (MA), 2013-2017

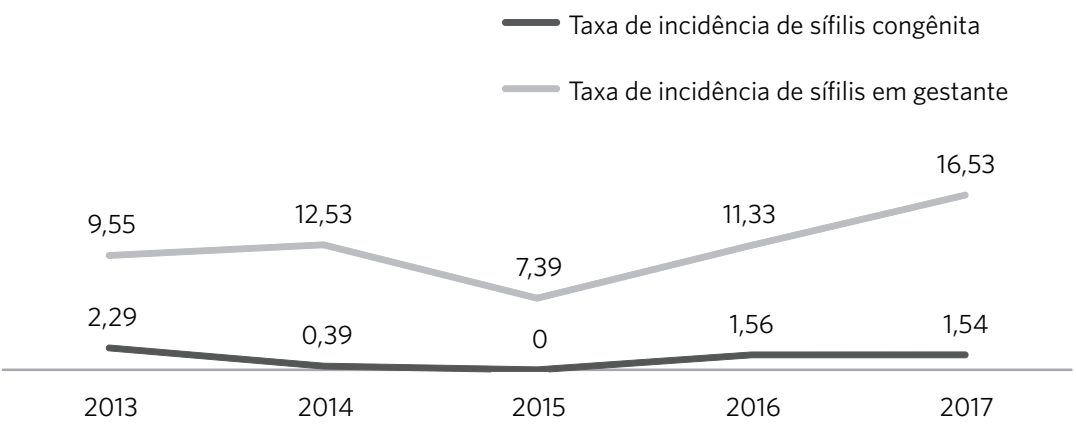

Fonte: Maranhão10,11

Nos casos de sífilis gestacional, em relação às características sociodemográficas, verificaram-se as maiores prevalências em mulheres na faixa etária de 20 a 24 anos de idade, que se autodeclararam pardas, donas de casa, residentes na zona urbana e que tinham menos de oito anos de estudos (tabela 1).

A frequência do diagnóstico da infecção no terceiro trimestre gestacional foi identificada em $46,3 \%(n=69)$ das gestantes, sendo que $71,1 \%(n=106)$ apresentaram sífilis primária e $45,6 \%(n=68)$ realizaram tratamento com penicilina G benzatina 2.400.000 UI, observando-se que, para o diagnóstico, 79,9\% ( $\mathrm{n}=119)$ e $45,6 \%(n=68)$ das gestantes foram reagentes para os testes não treponêmico e treponêmico, respectivamente, identificando que $49,7 \%$ ( $\mathrm{n}=74)$ apresentaram titulação $>1: 8$. Observouse a não realização do tratamento em 59,1\% $(n=88)$ dos parceiros sexuais. O motivo do não tratamento não foi preenchido em $53 \%$ ( $n=79)$ das fichas de investigação, e em 26,8\% $(n=40)$, esse campo apresentou-se em branco, sem resposta (tabela 1).

Tabela 1. Características sociodemográficas, clínicas e dos parceiros das gestantes sororreagentes para sífilis em Caxias (MA), 2013 a 2017

\begin{tabular}{lrr}
\hline Variáveis (N=149) & $\mathbf{n}$ & $\%$ \\
\hline Faixa etária (em anos) & & \\
\hline 15 a 19 & 40 & 26,8 \\
20 a 24 & 45 & 30,2 \\
25 a 29 & 32 & 21,5 \\
30 a 34 & 16 & 10,7 \\
35 a 39 & 14 & 9,5 \\
$\geq 40$ & 2 & 1,3 \\
\hline Raça/cor & & 8,1 \\
\hline Branca & 12 & 1,3 \\
Indígena & 2 & 66,4 \\
Parda & 99 & 2 \\
\hline
\end{tabular}


Tabela 1. (cont.)

\begin{tabular}{|c|c|c|}
\hline Variáveis ( $N=149)$ & $\mathrm{n}$ & $\%$ \\
\hline \multicolumn{3}{|l|}{ Raça/cor } \\
\hline Preta & 23 & 15,4 \\
\hline Ignorado & 13 & 8,8 \\
\hline \multicolumn{3}{|l|}{ Escolaridade } \\
\hline Analfabeto & 4 & 2,7 \\
\hline$\leq 8$ anos & 68 & 45,7 \\
\hline$>8$ anos & 56 & 37,6 \\
\hline Ignorado & 21 & 14 \\
\hline \multicolumn{3}{|l|}{ Ocupação } \\
\hline Dona de casa & 64 & 42,9 \\
\hline Lavradora & 21 & 14,1 \\
\hline Estudante & 10 & 6,7 \\
\hline Doméstica & 4 & 2,7 \\
\hline Desempregada & 2 & 1,3 \\
\hline Outras ocupações & 5 & 3,5 \\
\hline Ignorada & 43 & 28,8 \\
\hline \multicolumn{3}{|l|}{ Localização } \\
\hline Urbana & 115 & 77,2 \\
\hline Rural & 27 & 18,1 \\
\hline Ignorado & 7 & 4,7 \\
\hline
\end{tabular}

Idade gestacional a notificação (Trimestre)

\begin{tabular}{lrr}
\hline Primeiro & 22 & 14,8 \\
Segundo & 45 & 30,2 \\
Terceiro & 69 & 46,3 \\
Idade gestacional ignorada & 13 & 8,7 \\
\hline
\end{tabular}

\section{Classificação clínica}

\begin{tabular}{lrr}
\hline Primária & 106 & 71,1 \\
Secundária & 3 & 2 \\
Terciária & 3 & 2 \\
Latente & 4,8 \\
lgnorado & 33 & 22,1 \\
\hline Realizou teste treponêmico no pré-natal & 68 & 45,6 \\
\hline Reagente & 6 & 4,1 \\
Não reagente & 54 & 36,2 \\
Não realizado & 21 & 14,1 \\
lgnorado & 119 & 79,9 \\
\hline Realizou teste não treponêmico no pré-natal & 10 & 6,7 \\
\hline Reagente & 60 \\
Não reagente & & \\
\hline
\end{tabular}


Tabela 1. (cont.)

Variáveis ( $\mathrm{N}=149)$

$\mathrm{n}$

$\%$

Realizou teste não treponêmico no pré-natal

Não realizado

9

Ignorado

11

Tratamento prescrito à gestante

Penicilina G benzatina 2.400.000 UI

$68 \quad 45,6$

Penicilina G benzatina 4.800.000 UI

Penicilina G benzatina 7.200.000 UI

$20 \quad 13,5$

Outro esquema

$24 \quad 16,1$

Não realizado

Tratamento do parceiro sexual

\begin{tabular}{llr}
\hline Sim & 37 & 24,8 \\
Não & 88 & 59,1 \\
Ignorado & 24 & 16,1 \\
\hline
\end{tabular}

Esquema de tratamento prescrito ao parceiro

Penicilina G benzatina 2.400.000 UI

Penicilina G benzatina 4.800.000 UI

$22 \quad 14,8$

Penicilina G benzatina 7.200.000 UI

12

Outro esquema

96

Não realizado

Ignorado

Motivo para o não tratamento do parceiro

Parceiro não teve mais contato com a gestante

\begin{tabular}{rr}
8 & 5,4 \\
4 & 2,7 \\
8 & 5,4 \\
1 & 0,7 \\
9 & 6 \\
79 & 53 \\
40 & 26,8 \\
\hline
\end{tabular}

Parceiro não foi comunicado/convocado à US* para tratamento

Parceiro foi comunicado/convocado à US* para tratamento, mas não compareceu

Parceiro foi comunicado/convocado à US*, mas recusou o tratamento

Parceiro com sorologia não reagente

Outro motivo

Fonte: Maranhão10

*US: Unidade de Saúde.

Nos casos de sífilis congênita, a caracterização do perfil materno apontou a prevalência de $27,8 \%(n=5)$ das mães com idades entre 20 e 24 anos, $61,7 \%$ ( $n=11)$ eram pardas e $38,9 \%$ $(\mathrm{n}=7)$ tinham menos de oito anos de estudos, constatando-se que $83,3 \%(n=15)$ tiveram o acompanhamento pré-natal e que $33,3 \%(n=6)$ foram diagnosticadas durante o trabalho de parto e/ou curetagem. O tratamento realizado nas mães das crianças notificadas com sífilis congênita foi preenchido como adequado em $72,2 \%(\mathrm{n}=13)$ das fichas de notificação, mas o tratamento não foi instituído em $77,8 \%(n=14)$ dos parceiros sexuais dessas mulheres, e 16,6\% $(n=3)$ dos casos foram preenchidos como informação ignorada (tabela 2). 
Tabela 2. Distribuição das características maternas dos casos notificados de sífilis congênita, entre 2013 e 2017, em Caxias (MA)

Variáveis $(\mathrm{N}=18)$

n

$\%$

Faixa etária (em anos)

\begin{tabular}{lrr}
\hline 15 a 19 & 4 & 22,2 \\
20 a 24 & 5 & 27,8 \\
25 a 29 & 4 & 22,2 \\
30 a 34 & 1 & 5,6 \\
35 a 39 & 2 & 11,1 \\
Ignorado & 2 & 11,1 \\
\hline Raça/cor & 3 & 16,7 \\
\hline Branca & 11 & 61,1 \\
Parda & 2 & 11,1 \\
Preta & 2 & 11,1 \\
Ignorado & 2 & 2 \\
\hline
\end{tabular}

\section{Escolaridade}

\begin{tabular}{lrr}
\hline$\leq 8$ anos & 7 & 38,9 \\
$>8$ anos & 5 & 27,8 \\
Ignorado & 5 & 27,8 \\
Não se aplica & 1 & 5,5 \\
\hline
\end{tabular}

\section{Realizou pré-natal}

\begin{tabular}{lrr}
\hline Sim & 15 & 83,3 \\
Não & 2 & 11,1 \\
Ignorado & 1 & 5,6 \\
\hline
\end{tabular}

Momento do diagnóstico de sífilis materna

\begin{tabular}{lrr}
\hline Durante o pré-natal & 4 & 22,2 \\
No momento do parto/curetagem & 6 & 33,3 \\
Após o parto & 5 & 27,8 \\
Não realizado & 1 & 5,6 \\
lgnorado & 2 & 11,1 \\
\hline
\end{tabular}

Tratamento das gestantes

\begin{tabular}{lrr}
\hline Adequado & 13 & 72,2 \\
Inadequado & 1 & 5,6 \\
Ignorado & 4 & 22,2 \\
\hline Tratamento dos parceiros das gestantes & 1 & 5,6 \\
\hline Sim & 14 & 77,8 \\
Não & 3 & 16,6 \\
\hline Ignorado & 3 & 14 \\
\hline
\end{tabular}

Fonte: Maranhãon! 
A infecção congênita foi prevalente em $77,8 \%(\mathrm{n}=14)$ dos recém-nascidos com idades entre 1 e 28 dias de vida, $61,1 \%$ ( $n=11)$ do sexo masculino e $55,6 \%(n=10)$ da raça parda, apresentando-se na forma clínica assintomática em 55,5\% ( $\mathrm{n}=10)$ das crianças, com $61 \%$ $(\mathrm{n}=11)$ reagentes para o teste não treponêmico realizado no sangue periférico. A penicilina G cristalina de 100.000 a $150.000 \mathrm{UI} / \mathrm{kg} / \mathrm{dia}$ foi o tratamento instituído em $33,3 \%(n=6)$, e, no que tange à evolução dos casos, $83,3 \%$ $(n=15)$ sobreviveram. No entanto, 5,6\% ( $n=1)$ evoluíram com natimortalidade (tabela 3 ).

Tabela 3. Distribuição das características sociais, assistenciais e diagnósticas relativas aos casos de crianças notificados entre 2013 e 2017

\begin{tabular}{|c|c|c|}
\hline Variáveis $(\mathrm{N}=18)$ & $\mathrm{n}$ & $\%$ \\
\hline \multicolumn{3}{|l|}{ Idade } \\
\hline $1 \mathrm{a}<28$ dias & 14 & 77,8 \\
\hline 29 dias a $\leq 1$ ano & 1 & 5,6 \\
\hline$>1$ ano & 3 & 16,6 \\
\hline \multicolumn{3}{|l|}{ Sexo } \\
\hline Masculino & 11 & 61,1 \\
\hline Feminino & 7 & 38,9 \\
\hline \multicolumn{3}{|l|}{ Raça/cor } \\
\hline Branca & 1 & 16,7 \\
\hline Parda & 10 & 55,6 \\
\hline Ignorado & 5 & 27,7 \\
\hline \multicolumn{3}{|c|}{ Presença de sinais e sintomas } \\
\hline Assintomático & 10 & 55,5 \\
\hline Sintomático & 1 & 5,6 \\
\hline Ignorado & 7 & 38,9 \\
\hline \multicolumn{3}{|c|}{ Teste não treponêmico - Sangue periférico } \\
\hline Reagente & 11 & 61 \\
\hline Não reagente & 1 & 5,6 \\
\hline Não se aplica & 3 & 16,7 \\
\hline Ignorado & 3 & 16,7 \\
\hline \multicolumn{3}{|c|}{ Teste não treponêmico - Líquor } \\
\hline Não realizado & 9 & 50 \\
\hline Ignorado & 9 & 50 \\
\hline \multicolumn{3}{|c|}{ Radiografia de ossos longos } \\
\hline Com alteração & 1 & 5,9 \\
\hline Não realizado & 7 & 41,2 \\
\hline Ignorado & 9 & 52,9 \\
\hline
\end{tabular}


Tabela 3. (cont.)

\begin{tabular}{|c|c|c|}
\hline Variáveis $(\mathrm{N}=18)$ & n & $\%$ \\
\hline \multicolumn{3}{|l|}{ Esquema de tratamento } \\
\hline Penicilina G cristalina 100.000 a $150.000 \mathrm{UI} / \mathrm{kg} / \mathrm{dia}$ - 10 dias & 6 & 33,3 \\
\hline Penicilina G procaína $50.000 \mathrm{UI} / \mathrm{kg} / \mathrm{dia}$ - 10 dias & 1 & 5,6 \\
\hline Penicilina G benzatina $50.000 \mathrm{UI} / \mathrm{kg} / \mathrm{dia}$ & - & - \\
\hline Outro esquema & 6 & 33,3 \\
\hline Ignorado & 5 & 27,8 \\
\hline \multicolumn{3}{|l|}{ Evolução do caso } \\
\hline Vivo & 15 & 83,3 \\
\hline Natimorto & 1 & 5,6 \\
\hline Ignorado & 2 & 11,1 \\
\hline
\end{tabular}

Fonte: Maranhãon.

A distribuição espacial dos focos de calor em relação à divisão municipal da região estudada mostrou predominância da ocorrência de sífilis gestacional e congênita, no período de 2013 a 2017, nas zonas Oeste e Leste, respectivamente, apresentando três pontos de concentração, com uma média de 9 a 11 casos por ponto. Os focos de calor na Região Central apresentaram baixa intensidade, evidenciando que essa área apresentou menores incidências para os agravos estudados (figura 1).

Figura 1. Distribuição espacial dos casos notificados de sífilis gestacional e congênita, na zona urbana de Caxias (MA), no período de 2013 a 2017

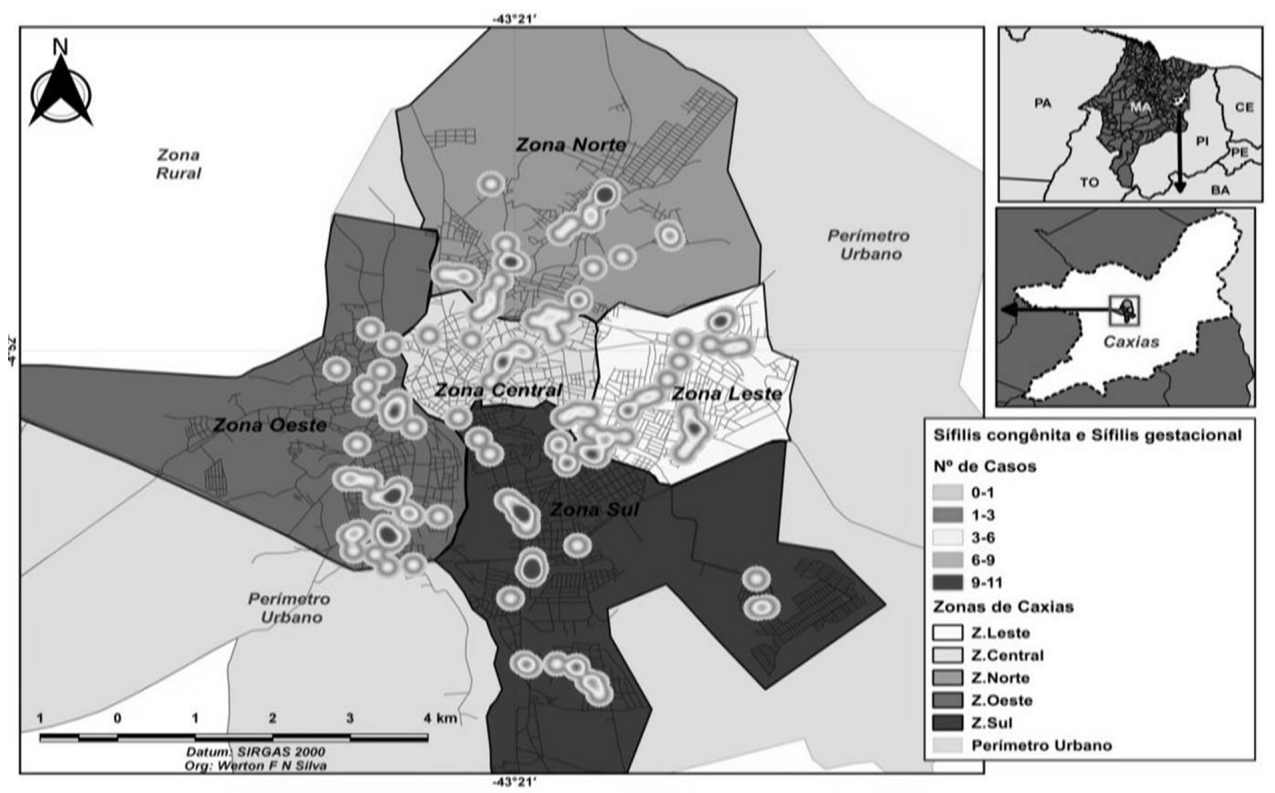




\section{Discussão}

O presente estudo apresenta os primeiros dados sobre sífilis gestacional e congênita no município de Caxias (MA), dimensiona sua magnitude como problema de saúde pública e reforça a importância de investimentos na assistência pré-natal e capacitação dos profissionais para o manejo correto da infecção.

A pesquisa evidenciou aumento da taxa de incidência da infecção em gestante, no município. Padrão semelhante foi encontrado em estudos conduzidos no estado do Piauí12 ${ }^{12}$ em Maringá (PR) ${ }^{\mathbf{1 3}}$, Palmas (TO) ${ }^{\mathbf{1 4}}$ e Santa Maria (RS) ${ }^{15}$. Uma análise dos dados do 'Boletim Epidemiológico de Sífilis’ revela que, no Brasil, houve aumento da taxa de incidência de sífilis gestacional, passando de 5 casos por mil nascidos vivos, em 2011, para 12,4 casos de sífilis em gestantes por mil nascidos vivos, em 2016 ${ }^{5}$. Pelo menos uma explicação para este achado precisa ser considerada: é possível que o aumento do número de casos de sífilis gestacional possa estar relacionado à não realização ou a inefetivas ações de educação em saúde ${ }^{6}$.

Destaca-se a maior prevalência da infecção em gestantes jovens (20 a 24 anos), pardas, com baixa escolaridade e donas de casa. Este achado corrobora os dados existentes na literatura, onde foi observado que a sífilis gestacional é mais incidente em mulheres na faixa etária de 20 a 35 anos ${ }^{15,17-19}$. Em contrapartida, um estudo realizado em Belo Horizonte (MG) encontrou a prevalência da infecção em gestante com idade inferior a 20 anos $^{20}$. A raça/cor parda, baixa escolaridade e o desenvolvimento de atividades sem remuneração são características prevalentes nas gestantes com sífilis, sendo encontrados resultados semelhantes em outros estudos120-23.

A baixa escolaridade está relacionada ao risco à saúde, uma vez que o menor acesso à informação interfere no entendimento sobre a importância dos cuidados com a saúde, principalmente no que se refere às medidas preventivas, dessa forma, prejudicando a interrupção na cadeia de transmissão ${ }^{23-24}$. Nesta pesquisa, observou-se que a maioria das gestantes tinha baixa escolaridade, o que se assemelha a uma pesquisa realizada em Santa Cruz $(\mathrm{RN})^{24}$ e em outras regiões do País ${ }^{10,25}$.

Identificou-se uma elevada frequência de diagnósticos da sífilis no terceiro trimestre de gestação. O diagnóstico da infecção no terceiro trimestre gestacional é considerado tardio e pode estar relacionado ao início tardio do pré-natal nas gestantes, bem como às baixas sensibilidade e efetividade da assistência pré-natal oferecida. Resultados semelhantes a esse foram encontrados em outros estudos ${ }^{12,14}$. Em dissonância com esses dados, um estudo realizado no Japão evidenciou que $78 \%$ dos casos de sífilis em gestantes foram diagnosticados no primeiro trimestre de gestação ${ }^{26}$.

Os resultados apresentados evidenciaram um maior número de casos de gestantes com sífilis primária, ou seja, que estavam no primeiro estágio da doença. Estudo análogo, referente aos casos notificados de gestantes com sífilis no período de 2008 a 2011, no município de São Luís (MA), verificou que 47,6\% dos casos de sífilis gestacional se encontravam na fase primária da infecção ${ }^{22}$. A sífilis primária também foi prevalente na análise epidemiológica da sífilis gestacional no Brasil, entretanto, a observância do predomínio dessa fase clínica em grande parte do preenchimento das fichas pode estar relacionada à ausência de conhecimento dos profissionais sobre a infecção, ou seja, o preenchimento pode ter sido feito de maneira equivocada. De acordo com a fisiopatologia da infecção, a prevalência é da fase latente, então, a sífilis dificilmente é diagnóstica na fase primária 5 .

O tratamento não foi realizado em uma porcentagem significativa dos parceiros das gestantes notificadas com sífilis. Resultados semelhantes foram encontrados em estudo realizado em Fortaleza (CE) e Natal (RN), no qual se obteve achado convergente a esse. Respectivamente, $62,9 \%$ e 48,1\% dos parceiros das gestantes não realizaram o tratamento ${ }^{18,27}$. É importante salientar que, para ser considerado como adequado, o tratamento das gestantes 
com sífilis deve ser realizado com penicilina, de acordo com a classificação clínica da infecção, instituído até 30 dias antes do parto. E o tratamento do parceiro deve ser realizado concomitantemente ao da gestante ${ }^{\mathbf{2 3}}$.

No que tange à sífilis congênita, identificou-se nos resultados um decréscimo na taxa de detecção, no período do estudo. No entanto, apesar dessa redução significativa, mesmo estando abaixo da média nacional em todos os anos do estudo, as taxas de incidência de sífilis congênita se apresentaram alarmantes ${ }^{5}$. Resultado aposto a esse foi encontrado em um estudo realizado no estado do Paraná, no qual foi observada uma tendência crescente na taxa de detecção de sífilis congênita ${ }^{\mathbf{2 8}}$, assim como em outros estados do País, como Sergipe, Mato Grosso e Piauí 29,30-32. Em contrapartida, estudo realizado em outras localidades do estado do Maranhão encontrou resultados semelhantes aos de Caxias (MA), evidenciando que a redução no número de caso de sífilis congênita é uma tendência estadual6.

No presente estudo, houve o predomínio da sífilis congênita em crianças filhas de mães que realizaram o acompanhamento pré-natal. No entanto, o diagnóstico da infecção materna ocorreu, sobretudo, durante o parto ou a curetagem, o que se mostrou tardio e configurou uma falha na assistência pré-natal para o manejo correto da infecção no município.

A falha na assistência pré-natal interfere na realização de diagnóstico precoce e tratamento adequado e em tempo oportuno. Assim, o elevado número de casos de sífilis congênita pode ser explicado pela falta de implementação das orientações do Ministério da Saúde na atenção pré-natal ${ }^{33}$. A introdução de políticas públicas que promovam a capacitação dos profissionais de saúde é necessária, visando a habilitá-los para a assistência pré-natal adequada, assim preparando-os para a notificação e o manejo clínico da sífilis no período gravídico ${ }^{35}$.

Já o diagnóstico de sífilis congênita foi realizado predominantemente em casos recentes, assintomáticos e com evolução, em sua maioria, para a cura. Entretanto, ainda foi observada natimortalidade entre as crianças com sífilis. Corroborando esse achado, a pesquisa realizada no Paraná entre 2007 e 2013 também observou o predomínio da infecção como sífilis congênita recente e com evolução dos casos para a cura ${ }^{28}$.

O elevado número de campos ignorados nas fichas de notificação de sífilis gestacional e congênita encontrado neste estudo é preocupante, uma vez que todas as variáveis encontradas nas fichas de notificação servem para avaliar a situação do agravo, bem como a assistência à saúde prestada. O preenchimento adequado das fichas permite o acesso às informações dos pacientes, facilitando a avaliação, o planejamento e a instituição de medidas para a redução e o controle desse agravo ${ }^{27}$.

A análise espacial evidenciou o predomínio da sífilis gestacional na Zona Oeste e os casos de sífilis congênita na Zona Leste. Fato que chama a atenção é a ausência de casos de sífilis congênita na Zona Oeste, uma vez que esse agravo está intimamente relacionado à sífilis gestacional. Vale destacar que as regiões com maior número de casos são caracterizadas por uma população com baixa escolaridade, pouco poder aquisitivo e dispõem de áreas descobertas de assistência básica de saúde. A sífilis apresenta uma relação com a vulnerabilidade social, e a caracterização das regiões de maior ocorrência é fundamental para auxiliar os profissionais de saúde e gestores no planejamento de ações ${ }^{28}$. O aumento da taxa de incidência de sífilis gestacional e congênita em algumas regiões pode estar associado a melhores condições de acesso e atendimento nas unidades de saúde, consequentemente, melhor acesso aos métodos diagnósticos e maiores taxas de notificações ${ }^{35}$.

O presente estudo teve por limitação a utilização de dados secundários, o que o torna passível a falhas de preenchimento e/ou incompletude de informações nas fichas de notificação, o que interfere diretamente na divulgação de informações que retratam de maneira real o perfil e o manejo dos casos de sífilis gestacional e congênita no município. 


\section{Conclusões}

Em suma, evidenciou-se um crescimento significativo de sífilis gestacional, caracterizando a infecção em gestantes jovens, de baixa escolaridade, donas de casa e que residem na zona urbana, revelando a fragilidade da assistência pré-natal prestada às gestantes, através do diagnóstico tardio, tratamento inadequado e da não realização do tratamento do parceiro, sendo estes, pontos fundamentais para evitar a transmissão vertical.

A taxa de detecção da sífilis congênita, ao contrário da sífilis gestacional, apresentou um declínio significativo, com a infecção predominante em crianças no período neonatal, do sexo masculino e pardas. E a distribuição espacial indicou prevalência dessas doenças nas regiões periféricas do município.

Observou-se fragilidade no preenchimento

\section{Referências}

1. Campos ALA, Araújo MAL, Melo SP, et al. Epide-

2. Soares LG, Zarpellon B, Soares LG, et al. Sífilis ges-

3. Carvalho IS, Brito RS. Sífilis congênita no Rio Granand Contributor ID). das fichas de investigação, o que comprometeu o diagnóstico da real situação desses agravos. Isto torna necessária uma educação permanente para os profissionais, a fim de qualificar a vigilância da sífilis gestacional e congênita, com o intuito de instituir identificação precoce, tratamento oportuno e acompanhamento efetivo.

\section{Colaboradores}

Conceição HN (0000-0001-6035-8280)*, contribuiu substancialmente para a concepção, o planejamento, a análise e a interpretação dos dados. Câmara JT (0000-0002-8312-1697)* contribuiu substancialmente para o planejamento, a análise e a interpretação dos dados, e para a revisão crítica do conteúdo. Pereira BM (0000-0001-8541-4031)* contribuiu substancialmente para a revisão crítica do conteúdo. miologia da sífilis gestacional em Fortaleza, Ceará, Brasil: um agravo sem controle. Cad. Saúde Pública. 2010; (26):1747-1755. tacional e congênita: características maternas, neonatais e desfecho dos casos. Rev. Bras. Saude Mater. Infant. 2017; 17(4):781-789.

4. World Health Organization. Guidelines for the treatment of Treponema pallidum (syphilis). Geneva: WHO; 2016.

5. Brasil. Ministério da Saúde, Secretaria de Vigilância em Saúde. Boletim Epidemiológico de sífilis. Brasília, DF; 2017. (v. 48. n. 36).

6. Guimarães TA, Alencar LCR, Fonseca LMB, et al. Sífilis em gestantes e sífilis congênita no Maranhão. Arq. Cienc. Saúde. 2018; 25(2):24-30. de do Norte: estudo descritivo do período 2007-2010. Epidemiol. Serv. Saúde. 2014; 23(2):287-94.
7. Saraceni V, Domingues RMSM, VellozoV, et al. Vigi- 
lância da sífilis na gravidez. Epidemiol. Serv. Saúde. 2007; 16 (2):103-111.

8. Gordis L. Epidemiologia. 4. ed. São Paulo: Revinter; 2010.

9. Brasil. Conselho Nacional de Saúde. Resolução $n^{\circ}$ 466, de 12 de dezembro de 2012. Aprovar as seguintes diretrizes e normas regulamentadoras de pesquisas envolvendo seres humanos. Diário Oficial da União. 12 Dez 2012.

10. Maranhão. Secretaria Municipal de Saúde de Caxias. Sistema Nacional de Agravos de Notificação. Fichas de investigação de sífilis em gestante. Caxias: Prefeitura de Caxias; 2017.

11. Maranhão. Secretaria Municipal de Saúde de Caxias. Sistema Nacional de Agravos de Notificação. Fichas de notificação/investigação de sífilis congênita. Caxias: Prefeitura de Caxias; 2017.

12. Barbosa DRM, Almeida MGD, Silva AO, et al. Perfil epidemiológico dos casos de sífilis gestacional. Rev. Enfer. UFPE. 2017; 11(5):1867-1874.

13. Ramos MG, Boni SM. Prevalência da sífilis gestacional e congênita na população do município de Maringá-PR. Saúde Pesq. 2018; 11(3):517-526.

14. Cavalcante PAM, Pereira RBL, Castro JGD. Sífilis gestacional e congênita em Palmas, Tocantins, 20072014. Epidemiol. Serv. Saúde. 2017; 26(1):255-264.

15. Santos TD, Lock NC, Lnza SS, et al. Perfil da sífilis gestacional e congênita no município de Santa Maria-RS: vivências multidisciplinares para troca de saberes. Saúde (Santa Maria). 2016; 42(2):215-224.

16. Pires ACS, Oliveira DD, Rocha GMNM, et al. "Ocorrência de sífilis congênita e os principais fatores relacionados aos índices de transmissão da doença no Brasil da atualidade-Revisão de Literatura”. Rev. UNINGÁ. 2018; 19(1):58-64.

17. Magalhães DMS, Kawaguchi IAL, Dias A, et al. Sífi- lis materna e congênita: ainda um desafio. Cad. Saúde Pública. 2013; (29):1109-1120.

18. Cardoso ARP, Araújo MAL, Cavalcante MDS, et al. Análise dos casos de sífilis gestacional e congênita nos anos de 2008 a 2010 em Fortaleza, Ceará, Brasil. Ciênc. Saúde Colet. 2018; (23):563-574.

19. Araújo LRL, Silva VCC, Gouveia Filho OS, et al. Prevalência de sífilis gestacional e congênita no estado de Goiás, Brasil. FAINOR. 2016; 9(2)49-58.

20. Nonato SM, Melo APS, Guimarães MDC. Sífilis na gestação e fatores associados à sífilis congênita em Belo Horizonte-MG, 2010-2013. Epidemiol. Serv. Saúde. 2015 ; (24):681-694.

21. Aquino GT, Gonçalves HC. Perfil das mulheres portadoras de sífilis gestacional em Santa Catarina no ano de 2012. Arq. Catarinenses de Med. 2016; 44(4):7281.

22. Furtado MFS, Brasil GVDS, Aragão FBA, et al. Fatores epidemiológicos da sífilis em gestantes no município de São Luís-MA. Rev. UNINGÁ. 2018; 52(1):5155 .

23. Padovani C, Oliveira RR, Pelloso SM. Sífilis na gestação: associação das características maternas e perinatais em região do sul do Brasil. Rev. Latino-Am. Enfermagem. 2018; (26):1-10.

24. Cabral BTV, Dantas JC, Silva JA, et al. Sífilis em gestante e sífilis congênita: um estudo retrospectivo. Rev. Ciênc. Plural. 2017; 3(3):32-44.

25. Bottura BR, Matuda L, Rodrigues PSS, et al. Perfil epidemiológico da sífilis gestacional e congênita no Brasil - período de 2007 a 2016/Epidemiological profile of gestational and congenital syphilis in Brazil from 2007 to 2016. Arq Med Hosp Fac Cienc Med Santa Casa São Paulo. 2019; 64(2):69-75.

26. Suzuki S, Sekizawa A, Tanaka M, et al. Current status of syphilis in pregnant women in Japan. J. of Maternal-Fetal Neonatal Med. 2017; 30(23):2881-2883. 
27. Oliveira SIM. Notificações de sífilis gestacional e congênita: uma análise epidemiológica. [dissertação]. Mato Grosso do Sul: Universidade Federal do Rio Grande do Norte; 2016. 78 p.

28. Signor M, Spagnolo LML, Tomberg JO, et al. Spatial distribution and characterization of cases of congenital syphilis. J. Nursing UFPE. 2018; 12(2):398-406.

29. Cruz VC, Silva JR, Gois CFL, et al. Epidemiologia da sífilis em gestante e congênita em Sergipe. In: I Congresso Internacional de Enfermagem; 2017 maio 9-12; Tiradentes. Tiradentes: Universidade Tiradentes; 2017. p. 1-4.

30. Silva LCVG, Teodoro CCJ, Silva JK, et al. Perfil dos casos de sífilis congênita em um município do sul de Mato Grosso/Profile of the cases of syphilis in a municipality the South of Mato Grosso/Perfil de los casos de sífilis en un municipio del Sur de Mato Grosso. J. Health NPEPS [internet]. 2017; 2(2):380-390.

31. Rodrigues IM, Ribeiro MA, Albuquerque IMAN, et al. Perfil e distribuição espacial da sífilis congênita em Sobral-CE no período de 2007 a 2013. Ciênc. Saúde. 2018; 11(2):70-76.
32. Almeida PD, Araujo Filho ACA, Araújo AKL, et al. Análise epidemiológica da sífilis congênita no Piauí. Revista Interd. 2015; 8(1):62-70.

33. Gonçalves HC, Sousa TO, Sakae TM. Incidência de sífilis congênita no estado de Santa Catarina no ano de 2012. Arq. Catarinenses de Med. 2017; 46(2):15-25.

34. Suto CSS, Silva DL, Almeida EDS, et al. Assistência pré-natal a gestante com diagnóstico de sífilis. Rev Enferm Atenção Saúde. 2016; 5(2):18-33.

35. Teixeira MA, Santos PP, Santos PN, et al. Perfil epidemiológico e sociodemográfico das crianças infectadas por sífilis congênita no município de Jequié/ Bahia. Rev. Saúde.Com [ínternet]. 2015 [acesso em 2018 nov 20]; 11(3):303-313. Disponível em: http:// www.uesb.br/revista/rsc/vll/vlln3a07.pdf.

Recebido em 14/04/2019

Aprovado em 01/10/2019

Conflito de interesse: inexistente

Suporte financeiro: não houve 